\title{
Corneal biomechanical properties in hyperthyroidism and thyroid eye disease
}

\author{
M. Reza Razeghinejad ${ }^{1,2}$, Amir R. Farsiani1 ${ }^{1,3}$, Ramin Salout ${ }^{1,4}$, Naser Owji1, Masoumeh Masoumpour ${ }^{1}$, Alireza Kharmandar ${ }^{5}$, Mohammad Zamani ${ }^{4}$
}

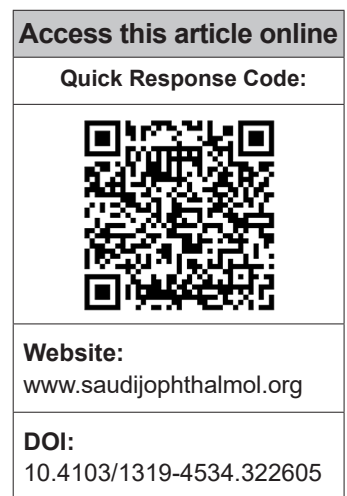

'Poostchi Ophthalmology Research Center, Department of Ophthalmology, School of Medicine, Shiraz University of Medical Sciences, Shiraz, Iran, ${ }^{2}$ Glaucoma Research Center, Wills Eye Hospital,

Philadelphia, PA, USA ${ }^{3}$ Department of Ophthalmology,

School of Medicine, Zanjan University of Medical sciences, Zanjan, ${ }^{4}$ Salouti Eye Research

Center, Salouti Eye Clinic,

Shiraz, ${ }^{5}$ Endocrine and Metabolism Research Center,

Shiraz University of Medical Sciences, Shiraz, Iran

Address for correspondence: Dr. Amir R. Farsiani,

Poostchi Ophthalmology Rese,

Poostchi Ophthalmology

Research Center, Zand Street,

Shiraz 713499744, Iran.

E-mail: amirrezafarsiani@ gmail.com

Submitted: 29-Jan-2019

Revised: $21-$ Apr-2020

Accepted: 06-Jul-2020

Published: 29-Jul-2021

\section{Abstract:}

PURPOSE: The purpose of this study is to compare the corneal biomechanical properties of hyperthyroids without ophthalmopathy (HWO), thyroid eye disease (TED), and euthyroid participants.

METHODS: In this prospective comparative study, one eye per patient included in the analysis and according to exclusion criteria, 38 eyes of 40 HWO patients, 31 eyes of 40 TED patients, and 150 eyes of 160 age- and sex-matched euthyroid participants were enrolled. All participants were evaluated by an endocrinologist and oculoplastic surgeon for evaluation of thyroid function and orbitopathy, respectively. Measurements of corneal biomechanical properties were performed using ocular response analyzer and Corvis ST tonometers.

RESULTS: In the HWO group, applanation-1 length (A1 L), applanation-2 velocity (A2V) $(P<0.001)$, and corneal resistant factor $(P=0.043)$ were higher than the control group and corneal hysteresis $(\mathrm{CH})$ was lower $(P=0.018)$. In the TED group, A1 L, A2V $(P<0.001)$, and highest concavity radius $(\mathrm{HCR})(P=0.027)$ were higher than the control group, and the $\mathrm{CH}(P=0.007)$ and highest concavity deformation amplitude $(\mathrm{HCDA})(P=0.001)$ were lower. Furthermore, the level HCDA in the TED group was lower than HWO group $(P=0.011)$. The level of upper scleral show and palpebral fissure had a negative correlation with $\mathrm{CH}$ and HCDA. The amount of exophthalmos level had a positive correlation with HCR.

CONCLUSION: Corneal biomechanical properties in the HWO and TED groups were different from the normal euthyroid individuals and may be taken into account in managing situation like glaucoma and refractive surgeries. Keywords:

Corneal biomechanics, corvis, ocular response analyzer, thyroid eye disease

\section{INTRODUCTION}

hyroid eye disease (TED) is an autoimmune disease. ${ }^{[1]}$ Ninety percent of all patients with TED have Graves' disease, and in about $6 \%$ of them the thyroid functions normally. TED is usually detected during the hyperactivity of the thyroid gland. ${ }^{[2,3]}$ It has two clinical phases of the inflammatory or active and the fibrotic or inactive. In the active phase (6-24 months), the levels of inflammatory cytokines are higher than in the inactive phase. Cytokines are considered as the markers of active TED and from among the causes of ocular surface changes in TED. ${ }^{[4,5]}$ Inflammatory cytokines stimulate keratocytes, leading to the production of matrix metalloproteinases as well as the subsequent corneal stromal destruction. ${ }^{[6]}$

This is an open access journal, and articles are distributed under the terms of the Creative Commons Attribution-NonCommercial-ShareAlike 4.0 License, which allows others to remix, tweak, and build upon the work non-commercially, as long as appropriate credit is given and the new creations are licensed under the identical terms.

For reprints contact: WKHLRPMedknow_reprints@wolterskluwer.com
In the inflammatory phase, the density of active keratocytes increases as against the inactive phase. ${ }^{[7]}$ Therefore, patients with TED may have different ocular biomechanical attributes, depending on the stage and the disease activity. In TED, a rise in extraocular muscle, adipose, and connective tissue volume leads to an increase in intraorbital pressure and orbital congestion, besides a secondary increase in episcleral venous pressure. Due to these orbital changes, $8.5 \%$ of TED patients develop ocular hypertension (OHT) and $2.5 \%$ of them develop glaucoma. The prevalence of OHT in TED patients is higher than the normal population. Although OHT is not preventable, routine ophthalmic examinations and diagnosis of these patients in the active phase of TED can prevent its progression to glaucoma. ${ }^{[8,9]}$ Reduced corneal hysteresis $(\mathrm{CH})$ is observed in glaucomatous eyes and is suggested as a risk

How to cite this article: Razeghinejad MR, Farsiani AR, Salouti R, Owji N, Masoumpour M, Kharmandar A, et al. Corneal biomechanical properties in hyperthyroidism and thyroid eye disease. Saudi J Ophthalmol 2020;34:251-5. 
factor for glaucoma progression. ${ }^{[10]}$ Given that TED patients are at the risk of glaucoma, the knowledge of corneal biomechanical properties may help define risk factors for glaucoma progression in this group of patients.

The ocular response analyzer (ORA; Reichert, Depew, NY, USA) is a noncontact tonometer that works based on applanation tonometry and measures the intraocular pressure (IOP) and corneal biomechanical properties. ${ }^{[11,12]}$ The Corvis ST (Oculus, Wetzlar, Germany) tonometer is also a noncontact tonometer equipped with a very high-speed camera that uses the Scheimpflug technology, assesses corneal deformation in response, and measures corneal biomechanical properties and IOP. ${ }^{[13-15]}$ Considering the effects of the thyroid function on corneal keratocytes and the high risk of glaucoma development in TED, this study was conducted to compare corneal biomechanical properties in hyperthyroids without ophthalmopathy (HWO), TED, and normal euthyroid participants.

\section{Methods}

In this prospective comparative study, a total of 240 patients were enrolled: 40 HWO, 40 TED, and 160 euthryroid. Informed consent was obtained from all participants, and the study was approved by the local Ethics Committee. Due to the significant effects of corneal thickness, after assessing all groups for this variable, eyes with corneal thickness exceeding 550 microns were excluded. Furthermore, only the data on the one eye of the patients were included for the statistical analysis. The analysis was performed on 38 eyes with HWO, 31 eyes with TED, and 150 eyes of age-and gender-matched euthyroid individuals with a normal eye examination.

Patients recently diagnosed with the Graves' disease at the endocrinology clinic were referred to the TED clinic for the evaluation of orbitopathy, and also they were categorized in TED or HWO groups based on the ophthalmic findings. All participants were referred to the same endocrinologist and oculoplastic surgeon for the examination of the thyroid function and orbitopathy, respectively. Given the fibrotic changes in TED, only patients diagnosed with TED in $<6$ months were enrolled. The control group was selected from among the participants with a normal eye examination who had attended a general ophthalmology clinic for routine eye examinations. The eye examination included slit-lamp biomicroscopy, Hertel exophthalmometery, IOP measurement, external eye examination, pupil and motility evaluation, and dilated fundoscopy. Thyroid function test was checked in all patients. Vision, inflammation, strabismus, and appearance/exposure criterion was used for TED staging. In the TED group, the following information was included: current smoking or history of smoking, marginal reflex distance 1 (MRD1), the upper and lower scleral show, palpebral fissure width, proptosis, lagophthalmos, and corneal findings.

The exclusion criteria included diabetes mellitus, hypertension, chronic kidney disease, glaucoma, IOP $>22 \mathrm{mmHg}$, corneal and retinal disorders, myopia $<-5.00$, hyperopia $>+3.00$, strabismus, and any previous ocular surgery.

All patients had ORA and Corvis ST tonometer measurements that were obtained randomly in one session. The tests were performed at an interval of $15 \mathrm{~min}$. The obtained information from the ORA was $\left(\mathrm{CH}\right.$, the difference between the $1^{\text {st }}$ and $2^{\text {nd }}$ applanations), Goldmann-correlated IOP (IOPg, the mean of these two applanations), corneal resistance factor (CRF), and corneal-compensated IOP (IOPcc). Only the data of the measurements with a waveform signal exceeding 3.5 were included in the statistical analysis, and the ones with values $<3.5$ were repeated. ${ }^{[16]}$

The Corvis ST parameters include applanation-1 time (time of the first applanation), applanation-2 time (time from start to the second applanation), highest concavity time (time of the highest displacement of the corneal apex), highest concavity deformation amplitude (HCDA: magnitude of the highest displacement of the corneal apex), first applanation-1 length (A1 L: the length of the flattened segment in the first applanation), second applanation-2 length (A2 L: the length of the flattened segment in the second applanation), applanation-1 and applanation-2 velocity (and A2V: corneal velocity of movement during two applanations), highest concavity peak distance (distance between bending points of the cornea at the highest concavity), highest concavity radius (HCR, central concave curvature at the highest concavity), central corneal thickness (CCT), and noncorrected IOP (IOPnct and corrected IOP based on corneal thickness (IOPpachy).

Student's $t$-test and analysis of variance and Mann-Whitney U-test and Kruskal-Wallis were used for the statistical analysis based on the distribution of the data. Bonferroni post hoc test was used if $P<0.05$. To identify a relationship, Pearson correlation coefficient, Spearman's rank correlation test, and multivariate regression analysis were used. $P<0.05$ was considered statistically significant. Statistical analysis was performed using the SPSS software version 23.0 (SPSS, Chicago, IL, USA).

\section{RESULTS}

Demographic data of all participants are presented in Table 1 and biomechanical properties in Table 2. In HWO group, A1 L, A2V, and $\mathrm{CRF}$ levels were higher than the control group, and $\mathrm{CH}$ level was lower. In TED group, the levels of A1 L, A2V, and HCR were higher, and $\mathrm{CH}$ and HCDA were lower than the control. Compared to the HWO, HCDA was lower in the TED group.

Table 1: Demographic data of all participants

\begin{tabular}{lcccc}
\hline Group & $\begin{array}{c}\text { HWO } \\
(\boldsymbol{n}=\mathbf{3 8} \text { eyes })\end{array}$ & $\begin{array}{c}\text { TED } \\
(\boldsymbol{n}=\mathbf{3 1} \text { eyes })\end{array}$ & $\begin{array}{c}\text { Control } \\
(\boldsymbol{n}=\mathbf{1 5 0} \text { eyes })\end{array}$ & $\boldsymbol{P}$ \\
\hline Age & $40.0 \pm 10.3$ & $35.0 \pm 10.0$ & $36.7 \pm 8.9$ & 0.066 \\
Gender & & & & \\
$\quad$ Male & $11(28.9 \%)$ & $13(41.9 \%)$ & $56(37.3 \%)$ & 0.501 \\
Female & $27(71.1 \%)$ & $18(58.1 \%)$ & $94(62.7 \%)$ & \\
\hline
\end{tabular}

$\mathrm{HWO}=$ Hyperthyroid without ophthalmopathy, TED=Thyroid eye disease 
The $\mathrm{CH}$ in smokers $(8.45 \pm 2.36)$ was lower than that of the nonsmokers $(9.72 \pm 2.16, P=0.024)$. Furthermore, $11(35.5 \%)$ euthyroid, $16(51.6 \%)$ hyperthyroid, and $4(12.9 \%)$ hypothyroid eyes were in this group. There was no statistically significant correlation between the thyroid function and corneal biomechanical variables.

Twenty-one (67.7\%) eyes of the TED group were in the inactive stage, and the severity of disease staging were as following: score $1(7,22.6 \%)$, score $2(1,3.23 \%)$, score $3(1,3.23 \%)$, and score $8(1,3.23 \%)$. Only A2 L in inactive TED (1.22 \pm 0.54$)$ was higher than those with active TED $(0.86 \pm 0.12, P=0.007)$.

In the TED group, Spearman's rank correlation test and multivariate regression analysis were used to identify a relationship between the clinical finding of TED and corneal biomechanical variables [Table 3]. There was no statistically significant correlation between TED activity score and lower scleral show with corneal biomechanical variables. Age and gender had no association with the corneal biomechanical properties in all groups.

In the TED group, CCT had an association with $\mathrm{CH}(P=0.007)$, CRF $(P=0.017)$, and IOPpachy $(P=0.003)$. An association was found between $\mathrm{CCT}$ and $\mathrm{CH}(P=0.018)$ and IOPpachy $(P=0.003)$ in the HWO group.

The IOPs measured by two devices are depicted in Table 4. Although the IOPg was similar in all groups, the IOPnct was highest in the TED followed by controls and HWO.

Table 2: Corneal biomechanical characteristics in all participants

\begin{tabular}{|c|c|c|c|c|c|c|}
\hline Variable & HWO (1) & TED (2) & Control (3) & $P(1$ vs. 2$)$ & $P(1$ vs. 3$)$ & $P(2$ vs. 3$)$ \\
\hline$\overline{\mathrm{CH}}$ & $2.15 \pm 9.42$ & $1.98 \pm 9.38$ & $1.98 \pm 10.24$ & 1.000 & 0.018 & 0.007 \\
\hline CRF & $2.11 \pm 10.77$ & $1.71 \pm 10.79$ & $1.79 \pm 10.10$ & 0.971 & 0.043 & 0.057 \\
\hline A1L & $0.30 \pm 2.02$ & $0.28 \pm 1.98$ & $0.09 \pm 1.78$ & 0.939 & $<0.001$ & $<0.001$ \\
\hline A1V & $0.02 \pm 0.14$ & $0.02 \pm 0.13$ & $0.03 \pm 0.13$ & 0.625 & 1.000 & 1.000 \\
\hline A2L & $0.30 \pm 1.00$ & $0.48 \pm 1.10$ & $0.40 \pm 1.04$ & 0.796 & 1.000 & 1.000 \\
\hline $\mathrm{A} 2 \mathrm{~V}$ & $0.25 \pm-0.64$ & $0.26 \pm-0.73$ & $0.23 \pm-0.93$ & 0.366 & $<0.001$ & $<0.001$ \\
\hline HCDA & $0.09 \pm 1.08$ & $0.10 \pm 1.01$ & $0.10 \pm 1.08$ & 0.011 & 1.000 & 0.001 \\
\hline HCPD & $1.01 \pm 4.41$ & $1.14 \pm 4.38$ & $0.97 \pm 4.53$ & 1.000 & 1.000 & 1.000 \\
\hline HCR & $0.79 \pm 7.54$ & $0.58 \pm 7.64$ & $0.37 \pm 7.38$ & 1.000 & 0.236 & 0.027 \\
\hline
\end{tabular}

Table 3: Relationship between the thyroid eye disease clinical findings and corneal biomechanical variables

\begin{tabular}{|c|c|c|c|c|c|c|c|c|}
\hline & \multicolumn{2}{|c|}{$\mathrm{MRD}_{1}$} & \multicolumn{2}{|c|}{ Upper scleral show } & \multicolumn{2}{|c|}{ Palpebral fissure } & \multicolumn{2}{|c|}{ Exophthalmos } \\
\hline & Coefficient & $P$ & Coefficient & $P$ & Coefficient & $P$ & Coefficient & $P$ \\
\hline$\overline{\mathrm{IOPg}}$ & 0.027 & 0.887 & 0.317 & 0.083 & 0.209 & 0.259 & $0.386^{*}$ & 0.032 \\
\hline IOPcc & 0.230 & 0.213 & $0.482 * *$ & 0.006 & $0.369^{*}$ & 0.041 & 0.170 & 0.359 \\
\hline $\mathrm{CH}$ & $-0.386^{*}$ & 0.032 & $-0.463 * *$ & 0.009 & $-0.423^{*}$ & 0.018 & 0.219 & 0.237 \\
\hline $\mathrm{CRF}$ & -0.317 & 0.083 & -0.319 & 0.081 & -0.326 & 0.073 & 0.335 & 0.066 \\
\hline IOPnct & 0.247 & 0.180 & $0.509^{* *}$ & 0.003 & $0.410^{*}$ & 0.022 & $0.492 * *$ & 0.005 \\
\hline IOPpachy & 0.199 & 0.283 & $0.396^{*}$ & 0.027 & $0.360^{*}$ & 0.047 & $0.378^{*}$ & 0.036 \\
\hline $\mathrm{A} 1 \mathrm{~L}$ & 0.52 & 0.781 & 0.133 & 0.475 & 0.102 & 0.585 & 0.224 & 0.226 \\
\hline A1V & -0.183 & 0.324 & $-0.443^{*}$ & 0.012 & -0.307 & 0.093 & -0.282 & 0.125 \\
\hline $\mathrm{A} 2 \mathrm{~L}$ & -0.101 & 0.590 & -0.018 & 0.924 & -0.036 & 0.847 & 0.118 & 0.529 \\
\hline $\mathrm{A} 2 \mathrm{~V}$ & -0.10 & 0.958 & 0.038 & 0.839 & 0.031 & 0.870 & 0.204 & 0.270 \\
\hline HCDA & -0.343 & 0.059 & $-0.598 * *$ & $<0.001$ & $-0.394 *$ & 0.028 & -0.277 & 0.132 \\
\hline HCPD & -0.047 & 0.802 & -0.310 & 0.089 & -0.133 & 0.475 & -0.293 & 0.110 \\
\hline $\mathrm{HCR}$ & -0.061 & 0.743 & 0.254 & 0.167 & 0.158 & 0.396 & $0.370^{*}$ & 0.040 \\
\hline
\end{tabular}

*Correlation is statistically significant at the 0.05 level, **Correlation is significant at the 0.01 level. $\mathrm{CH}=\mathrm{Corneal}$ hysteresis, $\mathrm{CRF}=\mathrm{Corneal}$ resistance factor, IOPcc=Corneal-compensated IOP, IOPg $=$ Goldmann-correlated IOP, A1L=A1 length, A2L=A 2 length, A1V=A velocity, A2V=A velocity, $\mathrm{HCPD}=$ Highest concavity peak distance, $\mathrm{HCR}=$ Highest concavity radius, HCDA=Highest concavity deformation amplitude, IOPnct=Noncorrected IOP, IOPpachy=Corrected IOP based on corneal thickness, MRD1=Margin reflex distant 1

Table 4: Intraocular pressures measured by corvis and ocular response analyzer in three groups

\begin{tabular}{lcccccc}
\hline & HWO $(\boldsymbol{n}=\mathbf{3 8}$ eyes) (1) & TED $(\boldsymbol{n}=\mathbf{3 1}$ eyes) (2) & Control $(\boldsymbol{n}=\mathbf{1 5 0}$ eyes) (3) & $\boldsymbol{P}$ (1 vs. 2) & $\boldsymbol{P}(\mathbf{1}$ vs. 3) & $\boldsymbol{P}(\mathbf{2}$ vs. 3) \\
\hline Central corneal thickness & $508.58 \pm 32.87$ & $516.71 \pm 26.13$ & $518.66 \pm 29.13$ & 0.846 & 0.181 & 1.000 \\
IOPg & $16.78 \pm 2.46$ & $17.03 \pm 2.68$ & $16.12 \pm 2.57$ & 1.000 & 0.214 \\
IOPcc & $17.18 \pm 2.93$ & $17.95 \pm 3.46$ & $16.58 \pm 2.20$ & 0.087 & 0.588 \\
IOPnct & $17.09 \pm 2.52$ & $18.07 \pm 2.92$ & $17.09 \pm 0.44$ & 0.031 & 1.000 & 0.011 \\
IOPpachy & $18.66 \pm 2.72$ & $19.42 \pm 3.32$ & $19.78 \pm 0.24$ & 0.195 & 0.001 & 0.855 \\
\hline
\end{tabular}

$\mathrm{HWO}=$ Hyperthyroid without ophthalmopathy, TED=Thyroid eye disease, IOPcc=Corneal-compensated IOP, IOPg=Goldmann-correlated IOP, IOPnct=Noncorrected IOP, IOPpachy=Corrected IOP based on corneal thickness 
The IOPpachy was lower in the HWO than TED and control groups. The maximum value for IOPcc was observed in the TED and the lowest in the controls. IOPcc and IOPpachy are claimed as cornea compensated IOP, but IOPcc values were lower than IOPpachy.

\section{Discussion}

Mechanical and inflammatory factors play a significant role in the ocular findings of TED and can change ocular and corneal biomechanical properties. The current study showed some differences in corneal biomechanical properties among the patients with HWO, TED, as well as the controls.

In $\mathrm{HWO}$ group, $\mathrm{A} 1 \mathrm{~L}, \mathrm{~A} 2 \mathrm{~V}$, and $\mathrm{CRF}$ levels were higher than the control group, and $\mathrm{CH}$ was lower. The levels of $\mathrm{A} 1 \mathrm{~L}, \mathrm{~A} 2 \mathrm{~V}$, and HCR in the TED group were higher than the control group, and the levels of $\mathrm{CH}$ and HCDA were lower. This results were similar to those of ORA-based studies in which the $\mathrm{CH}$ level was lower in patients with TED than the control subjects. ${ }^{[17-19]}$ The $\mathrm{CH}$ level was lower in ectatic corneal disorders, ${ }^{[20,21]}$ and the lower $\mathrm{CH}$ level was realized as an independent risk factor for the glaucoma progression. ${ }^{[22]}$ The inflammatory process in TED reduced the corneal damping ability ${ }^{[19]}$ and could cause changes to the $\mathrm{CH}$ and HCDA.

In ORA-based studies on normal individuals, the $\mathrm{CH}$ value was similar to that in smokers and nonsmokers; ${ }^{[23,24]}$ however, the $\mathrm{CH}$ value was lower in smokers in the current study. This could be related to the significant effect of smoking on the progression of TED and the inflammatory changes affecting $\mathrm{CH}$, not the direct effect of smoking on corneal biomechanical factors. ${ }^{[25]}$ Kilavuzoglu et al. compared corneal biomechanics in smokers and nonsmokers but found no difference between $\mathrm{CH}$ and $\mathrm{CRF}$. However, the $\mathrm{CH}$ value decreased with age and was less noticeable in the smoking group. ${ }^{[23]}$ The authors attributed this finding to the potential changes in the cornea's microstructure brought about by smoking and intensified by aging, besides the effects of smoking on the viscosity of the aged corneal tissue. Some of the TED patients may develop glaucoma and become more prone to glaucoma progression that can be aggravated by smoking, given the lower $\mathrm{CH}$ value.

In the current study, corneal biomechanical properties in the TED group were not affected by thyroid status. However, the effects of the thyroid gland status on corneal biomechanical properties were not considered in previous reports in TED. ${ }^{[17-19]}$

Although a negative relationship between $\mathrm{CH}$ and TED activity score has been reported previously, ${ }^{[17-19]}$ we did not find any statistically significant correlation between TED activity score and lower scleral show with corneal biomechanical properties. A2 L level among patients with inactive TED compared to the active TED was higher. In a study using Corvis ST in patients with TED, HCDA was lower in comparison to euthyroid individuals which is in line with our findings. ${ }^{[26]}$

The upper scleral show and palpebral fissure levels had a negative relationship with $\mathrm{CH}$ and HCDA. In addition, MRD1 level had a negative relationship with $\mathrm{CH}$. Exophthalmos levels had a positive correlation with HCR. A negative correlation between $\mathrm{CH}$ and amount of exophthalmos has been reported. ${ }^{[17-19]}$

In ORA-based studies on normal individuals, the $\mathrm{CH}$ level decreased with age. ${ }^{[27]}$ Besides, in Corvis ST-based studies on normal individuals, there was no correlation between gender and corneal biomechanical data, but the A1 L, A2V, HCR, and HCDA levels had a positive correlation with age. ${ }^{[28]}$ In the present research, the variable of age had no correlation with other corneal biomechanical properties in all groups.

Furthermore, $\mathrm{CCT}$ had a positive correlation with $\mathrm{CH}$ in the HWO group. Likewise, it had a positive correlation with $\mathrm{CH}$ and CRF in the TED group. These results were in line with the studies on healthy individuals. ${ }^{[29-31]}$ Although in the current work, no correlation was observed between, IOPcc and IOPnct with CCT, in a study on glaucoma patients, where these two factors were assessed, it was shown that all IOPs other than IOPcc were affected by CCT. ${ }^{[32]}$ As lower $\mathrm{CH}$ is correlated with the glaucoma progression, measuring the $\mathrm{CH}$ and IOPcc levels may be a valuable tool for the evaluation of glaucoma in patients with thyroid disorders.

The IOPg level was similar among the three groups; however, the IOPpachy level was lower in the HWO group than the TED and the control groups; moreover, the IOPcc level was highest in the TED group and lowest in the control group. IOPcc and IOPpachy were claimed as cornea compensated IOP, IOPcc was lower than IOPpachy. These findings were similar to the ORA-based studies, where the IOPcc level was higher in the TED group than the control group. ${ }^{[17-19]}$

One of the limitations of this study was the small sample size of the TED group that affected the evaluation of the effects of the disease severity on corneal biomechanical properties. However, measuring corneal biomechanical properties using the two machines could increase the validity of the results.

\section{Conclusions}

Corneal biomechanical characteristics in HWO and TED patients were different compared to normal subjects. Given the role of corneal biomechanical factors in glaucoma and keratorefractive surgeries, they may be taken into account in the management of these conditions in patients with HWO and TED.

\section{Financial support and sponsorship}

Nil.

\section{Conflicts of interest}

There are no conflicts of interest.

\section{REFERENCES}

1. Maheshwari R, Weis E. Thyroid associated orbitopathy. Indian J Ophthalmol 2012;60:87-93.

2. Bartley GB, Fatourechi V, Kadrmas EF, Jacobsen SJ, Ilstrup DM, 
Garrity JA, et al. Clinical features of graves' ophthalmopathy in an incidence cohort. Am J Ophthalmol 1996;121:284-90.

3. Versura P, Campos EC. The ocular surface in thyroid diseases. Curr Opin Allergy Clin Immunol 2010;10:486-92.

4. Huang D, Luo Q, Yang H, Mao Y. Changes of lacrimal gland and tear inflammatory cytokines in thyroid-associated ophthalmopathy. Invest Ophthalmol Vis Sci 2014;55:4935-43.

5. Huang $\mathrm{D}, \mathrm{Xu} \mathrm{N}$, Song $\mathrm{Y}$, Wang P, Yang H. Inflammatory cytokine profiles in the tears of thyroid-associated ophthalmopathy. Graefes Arch Clin Exp Ophthalmol 2012;250:619-25.

6. Sakimoto T, Ohnishi T, Ishimori A. Simultaneous study of matrix metalloproteinases, proinflammatory cytokines, and soluble cytokine receptors in the tears of noninfectious corneal ulcer patients. Graefes Arch Clin Exp Ophthalmol 2014;252:1451-6.

7. Villani E, Viola F, Sala R, Salvi M, Mapelli C, Currò N, et al. Corneal involvement in graves' orbitopathy: An in vivo confocal study. Invest Ophthalmol Vis Sci 2010;51:4574-8.

8. Behrouzi Z, Rabei HM, Azizi F, Daftarian N, Mehrabi Y, Ardeshiri M, et al. Prevalence of open-angle glaucoma, glaucoma suspect, and ocular hypertension in thyroid-related immune orbitopathy. J Glaucoma 2007; 16:358-62.

9. Cross JM, Girkin CA, Owsley C, McGwin G Jr. The association between thyroid problems and glaucoma. Br J Ophthalmol 2008;92:1503-5.

10. Wells AP, Garway-Heath DF, Poostchi A, Wong T, Chan KC, Sachdev N. Corneal hysteresis but not corneal thickness correlates with optic nerve surface compliance in glaucoma patients. Invest Ophthalmol Vis Sci. 2008;49:3262-8.

11. Piñero DP, Alcón N. Corneal biomechanics: A review. Clin Exp Optom 2015;98:107-16.

12. Dupps WJ Jr. Hysteresis: New mechanospeak for the ophthalmologist. J Cataract Refract Surg 2007;33:1499-501.

13. Hon Y, Lam AK. Corneal deformation measurement using scheimpflug noncontact tonometry. Optom Vis Sci 2013;90:e1-8.

14. Tian L, Huang YF, Wang LQ, Bai H, Wang Q, Jiang JJ, et al. Corneal biomechanical assessment using corneal visualization scheimpflug technology in keratoconic and normal eyes. J Ophthalmol 2014;2014: 147516.

15. Frings A, Linke SJ, Bauer EL, Druchkiv V, Katz T, Steinberg J, et al. Effects of laser in situ keratomileusis (LASIK) on corneal biomechanical measurements with the corvis ST tonometer. Clin Ophthalmol 2015;9:305-11.

16. Lam AK, Chen D, Tse J. The usefulness of waveform score from the ocular response analyzer. Optom Vis Sci 2010;87:195-9.

17. Karabulut GO, Kaynak P, Altan C, Ozturker C, Aksoy EF, Demirok A, et al. Corneal biomechanical properties in thyroid eye disease. Kaohsiung J Med Sci 2014;30:299 304
18. Zhang Y, She XX, Yu XJ, Chen LF, Shen LJ. [The corneal biomechanical properties of patients with graves' orbitopathy]. Zhonghua Yan Ke Za Zhi 2016;52:263-7.

19. Moghimi S, Safizadeh M, Mazloumi M, Hosseini H, Vahedian Z, Rajabi MT, et al. Evaluation of corneal biomechanical properties in patients with thyroid eye disease using ocular response analyzer. J Glaucoma 2016;25:269-73.

20. Ortiz D, Piñero D, Shabayek MH, Arnalich-Montiel F, Alió JL. Corneal biomechanical properties in normal, post-laser in situ keratomileusis, and keratoconic eyes. J Cataract Refract Surg 2007;33:1371-5.

21. Wolffsohn JS, Safeen S, Shah S, Laiquzzaman M. Changes of corneal biomechanics with keratoconus. Cornea 2012;31:849-54.

22. Medeiros FA, Meira-Freitas D, Lisboa R, Kuang TM, Zangwill LM, Weinreb $\mathrm{RN}$, et al. Corneal hysteresis as a risk factor for glaucoma progression: A prospective longitudinal study. Ophthalmology 2013;120:1533-40.

23. Kilavuzoglu AE, Celebi AR, Altiparmak UE, Cosar CB. The effect of smoking on corneal biomechanics. Curr Eye Res 2017;42:16-20.

24. Sayin N, Kara N, Yildirim Y, Altinkaynak H. A comparison of corneal biomechanical properties of chronic smokers and non-smokers using the ocular response analyzer. Adv Ophthalmol Vis Syst 2015;2:00027.

25. Thornton J, Kelly SP, Harrison RA, Edwards R. Cigarette smoking and thyroid eye disease: A systematic review. Eye (Lond) 2007;21:1135-45.

26. Vellara HR, Hart R, Gokul A, McGhee CNJ, Patel DV. In vivo ocular biomechanical compliance in thyroid eye disease. Br J Ophthalmol 2017;101:1076-9.

27. Kamiya K, Shimizu K, Ohmoto F. Effect of aging on corneal biomechanical parameters using the ocular response analyzer. J Refract Surg 2009;25:888-93.

28. Asaoka R, Nakakura S, Tabuchi H, Murata H, Nakao Y, Ihara N, et al. The relationship between corvis ST tonometry measured corneal parameters and intraocular pressure, corneal thickness and corneal curvature. PLoS One 2015;10:e0140385.

29. Wang W, He M, He H, Zhang C, Jin H, Zhong X, et al. Corneal biomechanical metrics of healthy Chinese adults using corvis ST. Cont Lens Anterior Eye 2017;40:97-103.

30. Shah S, Laiquzzaman M, Cunliffe I, Mantry S. The use of the reichert ocular response analyser to establish the relationship between ocular hysteresis, corneal resistance factor and central corneal thickness in normal eyes. Cont Lens Anterior Eye 2006;29:257-62.

31. Montard R, Kopito R, Touzeau O, Allouch C, Letaief I, Borderie V, et al. Ocular response analyzer: Feasibility study and correlation with normal eyes. J Fr Ophtalmol 2007;30:978 84.

32. Nakao Y, Kiuchi Y, Okimoto S. A comparison of the corrected intraocular pressure obtained by the corvis ST and reichert 7CR tonometers in glaucoma patients. PLoS One 2017;12:e0170206. 